\title{
LAS TECNOLOGÍAS DE LA INFORMACIÓN Y LA COMUNICACIÓN EN LOS PROCESOS DE ORIENTACIÓN PARA EL ESTUDIO A DISTANCIA
}

\author{
ICT IN THE ORIENTATION PROCESSES FOR DISTANCE LEARNING
}

\author{
Juan Ruiz Carrascosa* \\ Universidad de Jaén
}

\begin{abstract}
RESUMEN
El artículo que presentamos ofrece una investigación sobre la utilización de las TIC en el desarrollo de un plan de acogida para nuevos estudiantes de la Universidad Nacional de Educación a Distancia (UNED) y del programa de orientación desarrollado, que ofrecen información y asesoramiento para que los alumnos conozcan la metodología del estudio a distancia, así como los recursos virtuales disponibles para el desarrollo de los procesos de enseñanza y aprendizaje en educación superior. Presentamos los resultados de la evaluación de este programa, en el que han participado 140 estudiantes de 12 carreras diferentes y del curso de acceso para mayores de 25 años.
\end{abstract}

Palabras clave: Educación superior a distancia, TIC, plan de acogida, programa de orientación, técnicas de estudio, investigación evaluativa.

\begin{abstract}
This article reflects the results of a research about the use of ICT for the development of a welcoming plan for entering students in the public Spanish Open University (UNED). Within this plan, a guidance program has been carried out for students to become familiar with distance learning methodology, as well as the virtual resources available for the development of the teaching-learning processes in higher education.

Students have used both the computing facilities at the university and their own PCs at home in order to access the university's website. Attention has been paid to the competencies necessary for distance learning, the orientations for study planning, self-regulated study techniques and exam preparation.

The program evaluation results are provided. 140 students from 12 different degrees and from the access course for students over 25 years took part in the study. Two questionnaires were provided to

\footnotetext{
* Profesor Titular de la Universidad de Jaén, Área MIDE. Coordinador del COIE del Centro Asociado de la UNED en la provincia de Jaén. Líneas de investigación: Evaluación de programas y empleo de las TIC en educación.jruiz@ujaen
} 
measure the relevance of the virtual platform, the contents developed and the resources and materials used. Both a descriptive analysis and a correlation analysis among the different variables involved have been conducted. Conclusions and suggestions for the improvement of the guidance program are offered.

Key words: Distance higher education, ICT, reception plan, guidance program, study techniques.

\section{Introducción}

La adaptación de la oferta académica universitaria al Espacio Europeo de Educación Superior (EEES) es quizá el principal reto que han de afrontar nuestras universidades en los próximos años. El EEES supone la introducción de importantes cambios, tanto en la estructura de las titulaciones como en la metodología de enseñanza-aprendizaje y en los necesarios procesos de orientación a lo largo de la carrera. El enfoque metodológico subyacente al EEES promueve un aprendizaje centrado en el estudiante, con la puesta en práctica de metodologías activas de aprendizaje. Esto supone, tal como destaca Beltrán (1999, pág. 10) la emergencia de un nuevo paradigma educativo, "se trata de un paradigma que cambia sustancialmente el centro de gravedad y, en lugar de estar centrado en el profesor y la enseñanza, está centrado en el aprendizaje y la persona que aprende". Lo que importa ahora no es tanto la transmisión de conocimientos, sino ayudar a los alumnos a adquirir conocimientos, es decir, ayudar a aprender. Desde la perspectiva del desarrollo de los procesos de enseñanza y aprendizaje, se resalta la necesidad de incentivar el uso de métodos docentes más activos y de sistemas de autoaprendizaje por el alumnado, así como de potenciar la atención tutorial y el empleo de nuevos recursos tecnológicos en educación superior.

Para el desarrollo de estas nuevas metodologías, donde se promueve un aprendizaje activo y en gran medida autónomo, así como para la adquisición de competencias y habilidades orientadas al ejercicio profesional, el empleo de las tecnologías de la información y la comunicación (TIC) para dar soporte a los procesos de enseñanza-aprendizaje resulta imprescindible. Desde esta perspectiva, la Asociación de Universidades Europeas (2000) destaca la importancia de las nuevas tecnologías aplicadas a la enseñanza. En este sentido, quizás el ámbito más importante en el uso de las nuevas tecnologías de la información en educación superior se de en la enseñanza a distancia, tal como destaca Dirr (2007). Dentro del sistema de educación superior a distancia el uso de las TIC permite el acceso a diversas plataformas virtuales, tanto docentes como administrativas, así como a múltiples recursos para el estudio y la investigación. Estas nuevas posibilidades exigen procesos adecuados de orientación al alumnado, tanto al inicio como en el desarrollo de la carrera.

El empleo de las TIC en la orientación educativa cuenta con una trayectoria paralela al desarrollo de los recursos tecnológicos. Así, en una primera etapa los programas de orientación están vinculados al empleo de los ordenadores centrales, tal como indican Katz (1974), Butler y Dowsey (1978) y Wallis (1978).

Con la introducción de los ordenadores personales en las décadas de los años setenta y ochenta, se produce un incremento importante en el empleo de estos recursos tecnológicos en el ámbito educativo y de forma concreta en el campo de la orientación (Cairo y Kanner, 1984). Con el empleo de los microordenadores se desarrollan nuevos programas de orientación, poniéndose de manifiesto la ayuda importante que supone el uso del ordenador en las 
tareas de orientación (Crawley, 1992; Jackson, 1993; Repetto y Malik, 1998; Watts y Jackson, 2000).

La tercera etapa, en la que nos encontramos actualmente, comienza en los años noventa con la aparición de la World Wide Web (WWW) y su empleo en los centros educativos. El uso de Internet en educación superior ha puesto de manifiesto su utilidad tanto para el desarrollo de nuevos entornos de enseñanza y aprendizaje como para dar soporte a numerosos servicios y recursos para la orientación (Cebrián, 2004). Desde sitios Web se ofrece información académica para facilitar los procesos de elección de carrera y determinar los itinerarios de formación, obtener asesoramiento y personalizado on line, orientación para el desarrollo de técnicas de trabajo intelectual y empleo de recursos, así como ayuda para la búsqueda de empleo (Repetto y Guillamón, 2002). En el sistema superior de educación a distancia, la orientación a los alumnos al comienzo de la carrera es una tarea fundamental e ineludible. La multitud de recursos disponibles en las plataformas virtuales docentes y administrativas, así como las exigencias especiales que tiene el aprendizaje a distancia, requieren el desarrollo de procesos de orientación adecuados a este contexto de aprendizaje y a la tipología de su alumnado, que no asiste a clases presenciales y que en un porcentaje elevado hace compatible el estudio con el trabajo. En este ámbito se ha desarrollado el trabajo de investigación presentado en este artículo.

Dado que en nuestra investigación nos planteamos analizar el uso de las TIC en los procesos de orientación inicial a los estudiantes de nuevo ingreso, hemos de destacar la importancia que tienen las acciones desarrolladas por las universidades a través de jornadas de acogida y programas de orientación al inicio de la carrera. En el informe de evaluación del Plan Nacional de Evaluación de la Calidad de las Universidades (Ministerio de Educación, Cultura y Deporte, 2003) se destaca la necesidad de potenciar la orientación a los alumnos universitarios, sobre todo a los de nuevo ingreso. Para mejorar la atención a estos alumnos se propone potenciar las jornadas de acogida y la orientación inicial que les facilite su integración en la universidad. En este mismo sentido, la Unidad para la Calidad de las Universidades Andaluzas (2006) en su Informe Final del Plan Andaluz de Evaluación y Mejora de la Calidad de las Universidades, señala la necesidad de desarrollar y mejorar las políticas de información y asesoramiento sobre las titulaciones a los alumnos de nuevo ingreso. En este ámbito, en los últimos años se resalta la importancia que tienen los sistemas de apoyo y orientación a los universitarios de nuevo ingreso como criterios de calidad (Junquera, 2008 y Vieira, 2008).

La acción tutorial en la universidad, como ponen de relieve numerosos autores (Álvarez, 2002; Rodríguez, 2002; Del Rincón, 2005), debe desarrollarse desde el inicio de los estudios a fin de orientar a los alumnos para que puedan realizar una adecuada elección de sus itinerarios formativos y conozcan las exigencias y metodología de trabajo en educación superior.

La orientación académica al inicio de los estudios tiene una singular importancia en el sistema superior de educación a distancia, que se caracteriza por su metodología específica y el empleo de las TIC en los procesos de orientación y estudio. Éste es un campo donde son necesarias investigaciones como la realizada, con objeto de analizar la utilidad de los planes de acogida y los programas de orientación para el estudio autorregulado, así como el uso de las TIC en los mismos por parte de los alumnos de nuevo ingreso. 


\section{Método}

La investigación ha consistido en estudiar y evaluar el acceso y uso del Plan de Acogida para los alumnos de nuevo ingreso en la UNED y el programa de orientación desarrollado a principio de curso, dentro de este plan, para el conocimiento de los medios y recursos disponibles, así como de la metodología del estudio a distancia. Se ha utilizado una metodología descriptiva, a través de la técnica de encuesta, empleando dos cuestionarios construidos ad hoc para la evaluación por parte de los alumnos de los dos aspectos anteriormente señalados.

El Plan de Acogida de nuevos estudiantes de la UNED se desarrolla con la colaboración del Instituto Universitario de Educación a Distancia (IUED) y el Centro de Orientación, Información y Empleo (COIE). A través de este plan se pretende la preparación de los estudiantes para aprovechar las ventajas que ofrece la enseñanza a distancia en términos de flexibilidad y personalización del proceso de aprendizaje. Los objetivos de este plan son los siguientes:

- Asesorar a los estudiantes durante el proceso de matrícula.

- Facilitar su incorporación a la comunidad universitaria mediante:

- El conocimiento de los servicios de apoyo ofertados por la Universidad.

- La adquisición de competencias para llevar a cabo un aprendizaje autorregulado.

- El entrenamiento en técnicas de estudio.

- La utilización de Internet y el uso de los recursos virtuales.

El Plan de Acogida está disponible en la página Web de la UNED y ofrece una amplia información sobre los siguientes aspectos:

- Conoce tu Facultad/Escuela y tu programa de estudios.

- Conoce tu Centro Asociado.

- ¿Cómo estudiar en la UNED?

- Servicios generales de apoyo al estudiante.

- Cómo obtener más información.

Dentro de la información que se ofrece a los alumnos para estudiar a distancia hay que destacar las guías disponibles sobre los siguientes temas:

- Competencias necesarias para estudiar a distancia.

- Orientaciones para la planificación del estudio.

- Preparación de exámenes en la UNED.

- Técnicas de control de la ansiedad en los exámenes.

El acceso a toda esta información, disponible en la Web, es libre y voluntario por parte de los estudiantes y en muchos casos se ha podido comprobar que los alumnos que cursan primer año de carrera no tenían conocimiento de su existencia o bien no habían utilizado estos recursos de orientación. Ante esta situación, desde el COIE del centro asociado de la UNED de la provincia de Jaén se propone un programa de orientación para fomentar el acceso de los alumnos de nuevo ingreso al Plan de Acogida y a la vez poder asistir a las actividades semipresenciales que se ofertan a principio de curso para el desarrollo de este Plan. Este 
programa de orientación se lleva a cabo durante el primer mes del curso, con los alumnos matriculados en el curso de acceso a mayores de veinticinco años y primer curso de las diferentes carreras que se tutorizan en el centro. Los objetivos del programa son los siguientes:

- Dar a conocer el Plan de Acogida accesible en la página Web.

- Informar sobre los medios y recursos disponibles en el centro asociado.

- Conocer y utilizar la plataforma virtual docente y administrativa.

- Familiarizar al alumno con la metodología propia de la UNED.

- Dar a conocer las técnicas de estudio autorregulado.

- Entrenar al alumno en técnicas intelectuales.

- Informar al alumno sobre el sistema de evaluación en la UNED y entrenarle en los distintos tipos de pruebas presenciales y a distancia.

- Informar sobre técnicas para el control de la ansiedad en los exámenes.

Para el logro de estos objetivos se desarrollan los siguientes contenidos:

- La condición del estudiante a distancia.

- Estilos de aprendizaje.

- Técnicas de estudio.

- Técnicas metacognitivas.

- Elaboración de trabajos en grupo.

- Elaboración de proyectos de investigación.

- Desarrollo de la capacidad de crítica y valoración

- Metodología de estudio en la UNED y recursos disponibles.

- El sistema de evaluación en la UNED

- Control de estrés ante los exámenes

La metodología empleada se orienta hacia la gestión de la autonomía del alumno, combinando la actividad presencial y a distancia, procurando a través de la actividad reflexiva la construcción de un repertorio de actitudes y estrategias orientadas al estudio eficiente y al conocimiento de los recursos disponibles. Este programa tiene una duración de cuarenta horas, veinte de ellas son presenciales y se dedican a desarrollar los contenidos necesarios para al logro de los objetivos expuestos. Durante las veinte horas restantes los alumnos realizan las actividades propuestas en la fase presencial, fundamentalmente análisis y valoración de las guías para el estudio a distancia disponibles en la página Web, así como la realización de un trabajo práctico de planificación de sus asignaturas, a partir de todas las orientaciones y materiales que se les han proporcionado en la fase presencial.

En el desarrollo del programa de orientación se han utilizado las aulas de informática del centro y los ordenadores personales en casa, conectados a la Web de la Universidad. En el desarrollo del programa han participado personal de administración y servicios, profesores tutores y los coordinadores de TIC y COIE del centro.

Los instrumentos utilizados para la recogida de la información han sido los dos cuestionarios que aparecen al final de este artículo. El primero se ha dirigido a conocer el nivel de conocimientos previos y de expectativas de los alumnos y el segundo se ha centrado en la valoración del Plan de Acogida y del programa de orientación desarrollado. 


\section{Muestra}

La muestra, tal como se contempla en la Tabla 1, está compuesta por 140 alumnos matriculados en el curso de acceso para mayores de veinticinco años y en doce carreras diferentes, de las que se tutorizan en el centro asociado de la UNED en la provincia de Jaén. De los 140 alumnos de la muestra el $57 \%$ son hombres y el $43 \%$ mujeres. La edad media es de 36 años, con un valor mínimo de 21 años y máximo de 52 años, siendo la desviación típica para esta variable igual a 7,33. El 72\% indica tener trabajo y el $28 \%$ no. Estos datos se corresponden con el perfil típico del alumnado de la UNED, que compatibiliza estudio y trabajo y tiene una media de edad superior a la de los alumnos del sistema de enseñanza presencial.

TABLA 1: Distribución de la muestra por carreras (Frecuencias y porcentajes).

\begin{tabular}{|l|c|c|}
\hline \multicolumn{1}{|c|}{ Titulaciones } & Frecuencia & Porcentaje \\
\hline Curso de acceso & 48 & 34,3 \\
\hline Antropología & 5 & 3,6 \\
\hline Derecho & 20 & 14,3 \\
\hline Diplomatura en Empresariales & 11 & 7,9 \\
\hline Educación Social & 24 & 17,1 \\
\hline Filología Inglesa & 2 & 1,4 \\
\hline Historia & 2 & 1,4 \\
\hline Ingeniería Técnica & 5 & 3,6 \\
\hline Licenciatura Dirección y Administración Empresas & 4 & 2,9 \\
\hline Pedagogía & 3 & 2,1 \\
\hline Psicología & 5 & 3,6 \\
\hline Psicopedagogía & 5 & 3,6 \\
\hline Turismo & 6 & 4,3 \\
\hline Total & 140 & $\mathbf{1 0 0 , 0}$ \\
\hline
\end{tabular}

\section{Resultados}

Los alumnos que han tenido acceso al Plan de Acogida y han participado en el programa de orientación, han indicado en el 94\% de los casos no haber realizado con anterioridad este tipo de actividades, sólo el 6\% señala que ha realizado algún curso de técnicas de estudio anteriormente.

Al considerar si los alumnos disponen de Internet en casa, el 72\% de la muestra indica que tiene acceso desde su casa y el $28 \%$ no. Considerando la importancia de este recurso en 
la educación superior a distancia hemos analizado la relación entre tener trabajo y tener Internet en casa, mediante el análisis de la tabla de contingencia Internet-Trabajo (Tabla 2) y la prueba de chi cuadrado (Tabla 3). Como puede apreciarse, los valores obtenidos indican que existe una relación estadísticamente significativa $(\mathrm{p}<0.01)$ entre tener trabajo y disponer de Internet en casa y no tenerlo y estar en situación de desempleo.

TABLA 2: Tabla de contingencia Trabajo * Internet.

\begin{tabular}{|c|c|c|c|c|}
\cline { 3 - 4 } \multicolumn{2}{c|}{} & \multicolumn{2}{c|}{ Internet } & \multirow{2}{*}{$\begin{array}{c}\text { Total } \\
\text { NO }\end{array}$} \\
\cline { 3 - 5 } \multicolumn{2}{c|}{} & NO & sí & NO \\
\hline \multirow{2}{*}{ Trabajo } & NO & 27 & 12 & 39 \\
\cline { 2 - 5 } & sí & 12 & 89 & 101 \\
\hline \multicolumn{2}{|c|}{ Total } & $\mathbf{3 9}$ & $\mathbf{1 0 1}$ & $\mathbf{1 4 0}$ \\
\hline
\end{tabular}

TABLA 3: Pruebas de chi-cuadrado.

\begin{tabular}{|l|c|c|c|c|c|}
\cline { 2 - 6 } \multicolumn{1}{c|}{} & Valor & gl & $\begin{array}{c}\text { Sig. asintótica } \\
\text { (bilateral) }\end{array}$ & $\begin{array}{c}\text { Sig. exacta } \\
\text { (bilateral) }\end{array}$ & $\begin{array}{c}\text { Sig. exacta } \\
\text { (unilateral) }\end{array}$ \\
\hline Chi-cuadrado de Pearson & $46,046(\mathrm{~b})$ & 1 &, 000 & & \\
\hline Corrección por continuidad (a) & 43,236 & 1 &, 000 & & \\
\hline Razón de verosimilitudes & 43,864 & 1 &, 000 & &, 000 \\
\hline Estadístico exacto de Fisher & & & &, 000 & \\
\hline Asociación lineal por lineal & 45,717 & 1 &, 000 & & \\
\hline N de casos válidos & 140 & & & & \\
\hline
\end{tabular}

$\mathrm{Al}$ considerar las valoraciones que los alumnos realizan respecto a su interés sobre los contenidos del Plan de Acogida y sus conocimientos previos, se han obtenido los datos que se contemplan en las tablas 4 y 5 , donde se reflejan las puntuaciones medias (media aritmética en una escala de 1 a 5) y las correspondientes desviaciones típicas. El interés previo sobre los cinco módulos de contenidos es muy elevado en todos los casos, tal como puede comprobarse en la tabla 4, con valoraciones medias superiores a 4. Aunque con una pequeña diferencia, el mayor interés se manifiesta con relación a las técnicas de estudio (Media = $4,729)$ y el se menor se presenta respecto al conocimiento del sistema de educación a distancia: contexto y recursos $($ Media $=4,440)$.

Los resultados obtenidos sobre el nivel de conocimientos previos que tienen los alumnos en los aspectos reflejados en la Tabla 5 ponen de manifiesto que éste es bastante escaso en todos ellos. La plataforma virtual docente es el aspecto menos conocido por los alumnos $($ Media $=2,028)$ seguido del COIE $($ Media $=2,092)$. El funcionamiento administrativo de la UNED en general es el aspecto en el que los alumnos indican un mayor nivel de conocimiento previo (Media $=2,507$ ). Estos datos son muy importantes y refuerzan la necesidad del programa de orientación desarrollado a principio de curso. 
TABLA 4. Interés previo sobre módulos de contenidos.

\begin{tabular}{|l|c|c|}
\hline \multicolumn{1}{|c|}{ Módulos } & Media & Desv. tip \\
\hline 1. El sistema de Educación a Distancia. Contexto y recursos & 4,440 &, 702 \\
\hline 2. Estilos de aprendizaje & 4,671 &, 605 \\
\hline 4. Técnicas de estudio & 4,729 &, 632 \\
\hline 5. Técnicas metacognitivas & 4,607 &, 675 \\
\hline 4. El sistema de evaluación en la UNED & 4,628 &, 627 \\
\hline
\end{tabular}

TABLA 5. Nivel de conocimientos previos.

\begin{tabular}{|l|c|c|}
\hline \multicolumn{1}{|c|}{ Aspectos valorados } & Media & Desv. tip. \\
\hline 1. Funcionamiento administrativo de la UNED en general & 2,507 & 1,128 \\
\hline 2. Funcionamiento administrativo de la UNED en el Centro & 2,285 &, 976 \\
\hline 3. Medios y recursos disponibles en el Centro Asociado & 2,200 &, 953 \\
\hline 4. Plataforma virtual administrativa & 2,128 &, 973 \\
\hline 5. Plataforma virtual docente & 2,028 &, 988 \\
\hline 6. COIE & 2,092 & 1,010 \\
\hline
\end{tabular}

Los datos obtenidos en la valoración del Plan de Acogida ofrecen puntuaciones medias muy elevadas en la mayoría de los casos, tal como puede observarse en la Tabla 6. Las valoraciones medias obtenidas son superiores a 4, menos en el ítem que plantea el nivel de uso de las tutorías virtuales, donde la puntuación media obtenida es igual a 2,371. Este dato puede justificarse al haberse realizado la pregunta a alumnos de nuevo ingreso y a principio de curso. El valor de la desviación típica obtenido (Desv. Tip = 1,310), considerando el valor de la media, pone de manifiesto que hay notables diferencias por parte de los alumnos en la utilización de las tutorías virtuales.

Las valoraciones realizadas sobre el Programa de Orientación, desarrollado a principio de curso, son muy elevadas en todos los casos, tal como se aprecia en la tabla 7. El aspecto más valorado ha sido el curso de técnicas de estudio autorregulado, desarrollado de forma semipresencial, (Media $=4,985$ ) seguido de las orientaciones que se han dado durante la fase presencial para la preparación de exámenes y control de la ansiedad (Media $=4,900)$. La valoración global del programa de orientación desarrollado es muy elevada (Media $=$ 4,924). Esta puntuación es algo más elevada que la correspondiente a la valoración media del Plan de Acogida (Media = 4,814), habiéndose obtenido a través del coeficiente de Correlación de Pearson una correlación significativa $(\mathrm{p}<0.01)$ entre ambas variables $(\mathrm{r}=0,515)$. 
TABLA 6. Valoración del Plan de Acogida.

\begin{tabular}{|l|c|c|}
\hline \multicolumn{1}{|c|}{ Aspectos valorados } & Media & $\begin{array}{c}\text { Desv. } \\
\text { Tip }\end{array}$ \\
\hline $\begin{array}{l}\text { El Plan de Acogida responde a las necesidades iniciales de cualquier estudiante } \\
\text { novel en este sistema de enseñanza }\end{array}$ & 4,771 &, 421 \\
\hline Utiliza un lenguaje claro y preciso sobre los aspectos tratados & 4,828 &, 378 \\
\hline Ofrece información relevante sobre la tutoría & 4,850 &, 358 \\
\hline $\begin{array}{l}\text { Las técnicas de estudio propuestas favorecen el aprendizaje autorregulado de } \\
\text { forma idónea }\end{array}$ & 4,987 &, 119 \\
\hline Los ejemplos propuestos resultan útiles & 4,871 &, 335 \\
\hline Facilita el acceso a los recursos necesarios para la ampliación de la información & 4,700 &, 459 \\
\hline Expone con claridad las bases del trabajo en grupo a distancia & 4,628 &, 554 \\
\hline Incluye datos útiles de interés general sobre la evaluación & 4,900 &, 301 \\
\hline $\begin{array}{l}\text { Las orientaciones que se dan para la preparación de los exámenes facilitan la } \\
\text { planificación y desarrollo del aprendizaje }\end{array}$ & 4,871 &, 335 \\
\hline Los consejos para el control de la ansiedad ante los exámenes resultan útiles & 4,771 &, 421 \\
\hline $\begin{array}{l}\text { Presenta con claridad los diferentes medios de comunicación entre profesores y } \\
\text { alumnos }\end{array}$ & 4,700 & 4,75 \\
\hline Utilidad de este Plan para sus estudios & 4,885 &, 319 \\
\hline ¿En qué medida utiliza las tutorías virtuales? & 2,371 & 1,310 \\
\hline ¿Cuál es su nivel de expectativas respecto a la utilidad del Plan? & 4,692 &, 493 \\
\hline ¿Cuál es su valoración global del Plan de Acogida? & 4,814 &, 390 \\
\hline
\end{tabular}

Tabla 7. Valoración del Programa de Orientación.

\begin{tabular}{|l|c|c|}
\hline \multicolumn{1}{|c|}{ Aspectos valorados } & Media & Desv. tip \\
\hline Información presencial sobre plataforma virtual administrativa & 4,771 &, 421 \\
\hline Información presencial sobre plataforma virtual docente & 4,885 &, 319 \\
\hline Información sobre medios y recursos disponibles en el Centro & 4,850 &, 358 \\
\hline Curso de técnicas de estudio autorregulado & 4,985 &, 119 \\
\hline $\begin{array}{l}\text { Orientaciones para la preparación de exámenes y control de la ansiedad, } \\
\text { durante la fase presencial }\end{array}$ & 4,900 &, 330 \\
\hline Utilidad del trabajo práctico realizado & 4,871 &, 335 \\
\hline ¿Cuál es su valoración del programa de orientación? & 4,924 &, 232 \\
\hline
\end{tabular}




\section{Conclusiones}

Los resultados obtenidos a través de los cuestionarios empleados, así como toda la información que los alumnos han proporcionado sobre el Plan de Acogida y el desarrollo del programa de orientación que han llevado a cabo, indican un elevado nivel de expectativas respecto a este tipo de ayudas para el estudio a distancia, así como su utilidad en el inicio de los estudios. Los escasos conocimientos previos que tenían los alumnos sobre los aspectos que se han tratado justifican la inclusión de los módulos de contendidos que se desarrollan en el programa.

Las altas puntuaciones obtenidas en el cuestionario de valoración del Plan de Acogida y del programa de orientación ponen de manifiesto el logro de los objetivos propuestos, así como la utilidad de los contenidos que se han abordado y las actividades desarrolladas. A través del Plan de Acogida los alumnos han obtenido una mayor información sobre la plataforma virtual de la UNED, conociendo los recursos disponibles y familiarizándose con la metodología propia de esta Universidad. Mediante el programa de orientación se han entrenado en técnicas intelectuales y distintos sistemas de evaluación tanto presenciales como a distancia.

Con relación a la metodología empleada en las actividades llevadas a cabo, los alumnos han valorado de forma positiva la participación de profesores tutores junto a personal de administración y servicios y expertos en TIC, ya que esto ha permitido obtener una visión más completa de los aspectos docentes, administrativos y tecnológicos.

Como sugieren los alumnos en sus observaciones, las acciones de orientación que se han llevado a principio de curso tendrían que extenderse a todo el alumnado y desarrollarse a lo largo del curso. La oferta de orientación continua que ofrece el COIE puede dar respuesta a esta demanda.

La relación encontrada entre no tener Internet en casa y no tener trabajo, ha de tenerse en cuenta para poder proporcionar a los alumnos que se encuentran en esta situación las ayudas necesarias que faciliten el acceso a los recursos virtuales disponibles en la UNED, en general, y en el centro asociado en particular. El incremento del número de ordenadores en las aulas de extensión para el acceso del alumnado a las plataformas virtuales podría ser un primer paso en este sentido.

Para futuras investigaciones consideramos conveniente analizar la utilidad de las orientaciones recibidas por los alumnos a principio de curso para el desarrollo de sus tareas de aprendizaje, estimando en qué medida han utilizado y han resultado útiles las técnicas y estrategias estudiadas, así como los recursos disponibles para el estudio autorregulado. Asimismo, sería importante comprobar la utilidad de la información proporcionada respecto a la evaluación y la preparación de exámenes una vez finalizado el curso.

Igualmente sería interesante, en trabajos futuros, comparar el empleo de la metodología semipresencial, donde se combinan sesiones presenciales y uso autónomo de los recursos disponibles en la Web, con situaciones de acceso sólo a distancia a estos recursos para la orientación, y valorar las ventajas e inconvenientes de estos dos tipos de metodología para la orientación de los alumnos.

\section{Referencias Bibliográficas}

Álvarez, P. (2002). La función tutorial en la Universidad: una apuesta por la mejora de la calidad de la enseñanza. Madrid: EOS. 
Asociación de Universidades Europeas (2000). Evaluación formativa de la estrategia universitaria para las nuevas tecnologías aplicadas a la enseñanza y la formación. GUÍA DOC nº5. Ginebra: Asociación de Universidades Europeas.

Beltrán, J. (1999). “Aprender en la Universidad”. En J. Ruiz (Coord.): Aprender y enseñar en la Universidad. Iniciación a la docencia universitaria. (pp. 9-42). Jaén: Servicio de publicaciones de la Universidad de Jaén.

Butler, A. M. \& Dowsey, M. W. (1978). "Using a computer with a careers education model of guidance: the interactive Careers Guidance System”. Journal of Occupational Psychology, 51 (1), 57-68.

Cebrián, M. (2004). "Impacto de las tecnologías de la información y comunicación en la Universidad". Bordón Revista de Pedagogía, 56 (3 y 4), 587-600.

Cairo, P. C. \& Kanner, M. S. (1984). "Investigating the effects of compurerized approaches to counselor training". Counselor Education and Supervisión, 24 (2), 213-220.

Crawley, T. (1992). "Computer-Aided Career Guidance: An Investigation Involving an Artificial System". British Journal of Guidance \& Counselling, 20 (3), 344-352.

Del Rincón, B. (2005). Tutoría universitaria en la convergencia europea. Murcia: ICE Universidad.

Dirr, P. J. (2007). "Desarrollo social y educativo con las nuevas tecnologías". En F. Martínez y Mª P. Prendes (Coord.) Nuevas Tecnologías y Educación. (pp. 69-84). Madrid: Pearson Educación.

Jackson, C. (1993). "The Case for Diversity in Computer-Aided Career Guidance Systems: A Response to Watts". British Journal of Guidance \& Counselling, 21 (2), 189-196.

Junquera, S. (2008). Los sistemas de acogida y orientación a estudiantes en el programa VERIFICA. Documento electrónico: http.//www.urjc.es/aneca/Ponencias/14_S_JUNQUERA.pdf.

Ministerio de Educación, Cultura y Deporte (2003). Plan Nacional de Evaluación de la Calidad de las Universidades. Informe Global 1996-2000. Madrid: Secretaría General Técnica.

Katz, M. R. (1974). "Career decision-making: A computer-based System of Interactive Guidance and Information (SIGI)". In Measurement for Self-Understanding and Personal Development-Proceedings of the 1973 Invitational Conference on Testing Problems (pp. 43-69). Princeton, NJ: Educational Testing Service.

Repetto, E. y Guillamón, J. R. (2002). “Orientación y apoyo en la educación universitaria a distancia: estrategias organizativas y nuevas tecnologías aplicadas a la orientación a distancia”. En V. Álvarez y A. Lázaro (Coord.) Calidad de las universidades y orientación universitaria (pp. 347-367). Málaga: Aljibe.

Repetto, E. y Malik, B. (1998). "Nuevas tecnologías aplicadas a la orientación”. En R. Bisquerra (Coord.). Modelos de orientación e intervención psicopedagógica (pp. 363-374). Barcelona: Praxis.

Rodríguez, $\mathrm{M}^{\mathrm{a}}$ L. (2002). Hacia una nueva orientación universitaria: modelos integrados de acción tutorial, orientación curricular y construcción del proyecto profesional. Barcelona: Edicions de la Universitat.

Unidad para la Calidad de las Universidades Andaluzas (2006). Informe Final Andaluz. Plan andaluz de evaluación y mejora de la calidad de las universidades. Convocatoria 2002. Córdoba: UCUA.

Vieira, M. J. (2008). Criterios para la evaluación del sistema de apoyo y orientación al estudiante: revisión y propuestas. Revista de Educación, 345, 399-423.

Wallis, D. (1978). "Some pressing problems for research in vocational Guidance". Journal of Ocupational Psychology, 51, 7-18.

Watts, A. G. \& Jackson, C. (2000). "Networking a computer-aided guidance systemwithin higher education institutions: practice and potential". British Journal of Guidance \& Counselling, 28 (1), $55-73$. 


\section{ANEXO I \\ Cuestionario previo sobre el plan de acogida}

ALUMNO/A:

EDAD: MUJER:

HOMBRE:

TRABAJA: SI ; NO

DIRECCIÓN:

DISPONE DE ORDENADOR EN CASA CONECTADO A INTERNET:

TELÉFONO: CORREO ELECTRÓNICO:

ESTUDIOS QUE CURSA:

ESTUDIOS PREVIOS REALIZADOS:

FORMA DE ACCESO A LA UNED:

¿HA REALIZADO ANTERIORMENTE ALGUN CURSO DE TÉCNICAS DE ESTUDIO?

¿DÓNDE?

INDIQUE EN QUÉ MEDIDA LE INTERESAN CADA UNO DE LOS CINCO MÓDULOS.

Valore, poniendo una $X$, de 1 (poco) a 5 (mucho)

\begin{tabular}{|l|l|l|l|l|l|}
\hline \multicolumn{1}{|c|}{ Módulos } & $\mathbf{1}$ & $\mathbf{2}$ & $\mathbf{3}$ & $\mathbf{4}$ & $\mathbf{5}$ \\
\hline 1. El sistema de Educación a Distancia. Contexto y recursos & & & & & \\
\hline 2. Estilos de aprendizaje & & & & & \\
\hline 3. Técnicas de estudio & & & & & \\
\hline 4. Técnicas metacognitivas & & & & & \\
\hline 5. El sistema de evaluación en la UNED & & & \\
\hline
\end{tabular}

INDIQUE SU NIVEL DE CONOCIMIENTO DE CADA UNO DE LOS SIGUIENTES ASPECTOS:

\begin{tabular}{|l|l|l|l|l|l|}
\hline & $\mathbf{1}$ & $\mathbf{2}$ & $\mathbf{3}$ & $\mathbf{4}$ & $\mathbf{5}$ \\
\hline 1. Funcionamiento administrativo de la UNED en general & & & & & \\
\hline 2. Funcionamiento administrativo de la UNED en el Centro & & & & & \\
\hline 3. Medios y recursos disponibles en el Centro Asociado & & & & & \\
\hline 4. Plataforma virtual administrativa & & & & & \\
\hline 5. Plataforma virtual docente & & & & & \\
\hline 6. COIE & & & & & \\
\hline
\end{tabular}

\begin{tabular}{|l|l|l|l|l|l|}
\hline & $\mathbf{1}$ & $\mathbf{2}$ & $\mathbf{3}$ & $\mathbf{4}$ & $\mathbf{5}$ \\
\hline ¿Cuál es su nivel de expectativas respecto a la utilidad del Plan? & & & & & \\
\hline
\end{tabular}

OBSERVACIONES: 


\section{ANEXO II \\ Valoración del plan de acogida y programa de orientación para estudiantes nuevos de la UNED}

Carrera en que está matriculado:

Hombre: Mujer:

Trabaja: Si

; No ; Edad:

¿Tiene Internet en casa? Si ; No

Valore de 1 a 5 según esté más de acuerdo (5) o menos de acuerdo (1)

\begin{tabular}{|c|c|c|c|c|c|}
\hline Items & 1 & 2 & 3 & 4 & 5 \\
\hline $\begin{array}{l}\text { El Plan de Acogida responde a las necesidades iniciales de cualquier } \\
\text { estudiante novel en este sistema de enseñanza }\end{array}$ & & & & & \\
\hline Utiliza un lenguaje claro y preciso sobre los aspectos tratados & & & & & \\
\hline Ofrece información relevante sobre la tutoría & & & & & \\
\hline $\begin{array}{l}\text { Las técnicas de estudio propuestas favorecen el aprendizaje autorregulado } \\
\text { de forma idónea }\end{array}$ & & & & & \\
\hline Los ejemplos propuestos resultan útiles & & & & & \\
\hline $\begin{array}{l}\text { Facilita el acceso a los recursos necesarios para la ampliación de la } \\
\text { información }\end{array}$ & & & & & \\
\hline Expone con claridad las bases del trabajo en grupo a distancia & & & & & \\
\hline Incluye datos útiles de interés general sobre la evaluación & & & & & \\
\hline $\begin{array}{l}\text { Las orientaciones que se dan para la preparación de los exámenes facilitan } \\
\text { la planificación y desarrollo del aprendizaje }\end{array}$ & & & & & \\
\hline $\begin{array}{l}\text { Los consejos para el control de la ansiedad ante los exámenes resultan } \\
\text { útiles }\end{array}$ & & & & & \\
\hline $\begin{array}{l}\text { Presenta con claridad los diferentes medios de comunicación entre } \\
\text { profesores y alumnos }\end{array}$ & & & & & \\
\hline Utilidad de este Plan para sus estudios & & & & & \\
\hline ¿En qué medida utiliza las tutorías virtuales? & & & & & \\
\hline Información presencial sobre plataforma virtual administrativa & & & & & \\
\hline Información presencial sobre plataforma virtual docente & & & & & \\
\hline Información sobre medios y recursos disponibles en el Centro & & & & & \\
\hline Curso de técnicas de estudio autorregulado & & & & & \\
\hline $\begin{array}{l}\text { Orientaciones para la preparación de exámenes y control de la ansiedad, } \\
\text { durante la fase presencial }\end{array}$ & & & & & \\
\hline Utilidad del trabajo práctico realizado & & & & & \\
\hline
\end{tabular}




\begin{tabular}{|l|l|l|l|l|l|}
\hline \multicolumn{1}{|c|}{ Items } & $\mathbf{1}$ & $\mathbf{2}$ & $\mathbf{3}$ & $\mathbf{4}$ & $\mathbf{5}$ \\
\hline ¿Cuál es su valoración del programa de orientación? & & & & & \\
\hline ¿Cuál es su valoración global del Plan de Acogida? & & & & & \\
\hline
\end{tabular}

\section{OBSERVACIONES:}

¿Qué orientaciones considera más útiles en este Plan de Acogida? ¿Qué contenidos mejoraría?

¿Qué le añadiría?

Fecha de recepción: 02-02-2008

Fecha de revisión: 21-04-2009

Fecha de aceptación: 05-05-2009 